\title{
Avaliação de impacto do ciclo de vida: fatores de efeito para material particulado
}

Gabriela Roiko Cheli gabcheli@hotmail.com Universidade Tecnológica Federal do Paraná (UTFPR), Ponta Grossa, Paraná, Brasil.

\section{Gabriela Giusti}

gabriela.giusti@hotmail.com.br Universidade Federal de São Carlos (UFSCar), Sorocaba, São Paulo, Brasil.

\section{Letícia Yuriko Togawa}

leticia_yuriko@hotmail.com

Universidade Tecnológica Federal do

Paraná (UTFPR), Ponta Grossa, Paraná,

Brasil.

Diogo Aparecido Lopes Silva diogo.apls@ufscar.br

diogo.apls@ufscar.br
Universidade Federal de São Carlos (UFSCar), Sorocaba, São Paulo, Brasil.

\section{Yara de Souza Tadano} yaratadano@utfpr.edu.br Universidade Tecnológica Federal do Paraná (UTFPR), Ponta Grossa, Paraná Brasil.

\begin{abstract}
RESUMO
A atividade industrial é uma das principais causadoras de impactos ao meio ambiente, especialmente quanto à formação de material particulado ao ar. Uma forma de consideralos é usando a técnica de Avaliação do Ciclo de Vida, que objetiva analisar o ciclo de vida envolvido em um produto ou processo e associar os impactos causados em cada uma das etapas desse ciclo. Essa técnica tem quatro fases, sendo a fase de Avaliação de Impacto do Ciclo de Vida (AICV) abordada neste trabalho. Nela, relacionam-se os impactos com cada resultado da fase de Inventário do Ciclo de Vida, por meio de modelos de caracterização. Esses modelos objetivam calcular um fator de caracterização (FC), que irá mensurar o nível de impacto dentre as categorias de impacto existentes. Este trabalho tem como foco avaliar modelos de caracterização para a categoria de formação de material particulado, a fim de identificar os modelos mais adequados para serem utilizados no Brasil. A análise foi realizada dividindo o $\mathrm{FC}$ no fator de inalação e fator de efeito, sendo o último foco deste estudo. Três modelos foram analisados e os demais modelos existentes serão analisados posteriormente e avaliados quantitativamente, a fim de recomendar os mais adequados a serem aplicados no Brasil. Com isso, permite-se um avanço científico nas pesquisas de AICV no Brasil, além de possibilitar resultados mais próximos à realidade nacional.
\end{abstract}

Material particulado. Fatores de caracterização. Recomendação. Realidade Brasileira. AICV. 


\section{INTRODUÇÃO}

Diante do avanço no desenvolvimento urbano, há um aumento constante do número e tipos de fontes de emissão de poluição atmosférica. A Organização Mundial da Saúde (OMS) apresenta que $91 \%$ da população mundial vive em locais em que a qualidade do ar excede os seus limites padrões (OMS, 2019). A qualidade do ar depende do tipo de fonte poluidora, sendo a atividade industrial, uma das principais, unida aos veículos. Dentre os poluentes atmosféricos, encontra-se o material particulado (MP), foco deste estudo. Segundo a U.S.EPA (2019), o termo MP se refere a um conjunto de partículas sólidas e gotas de líquido encontradas no ar. As partículas de MP são extremamente pequenas, como pode ser observado na Figura 1, e influenciam negativamente nos impactos ambientais e na saúde humana. Elas são geralmente classificadas pelo seu tamanho, sendo mais prejudiciais à saúde, aquelas com diâmetro aerodinâmico menor que 10 micrometros $\left(\mathrm{MP}_{10}\right)$ e, principalmente, aquelas com diâmetro aerodinâmico menor que 2,5 micrometros $\left(\mathrm{MP}_{2,5}\right)$.

Figura 1 - Comparação do tamanho do material particulado com fio de cabelo e grão de areia

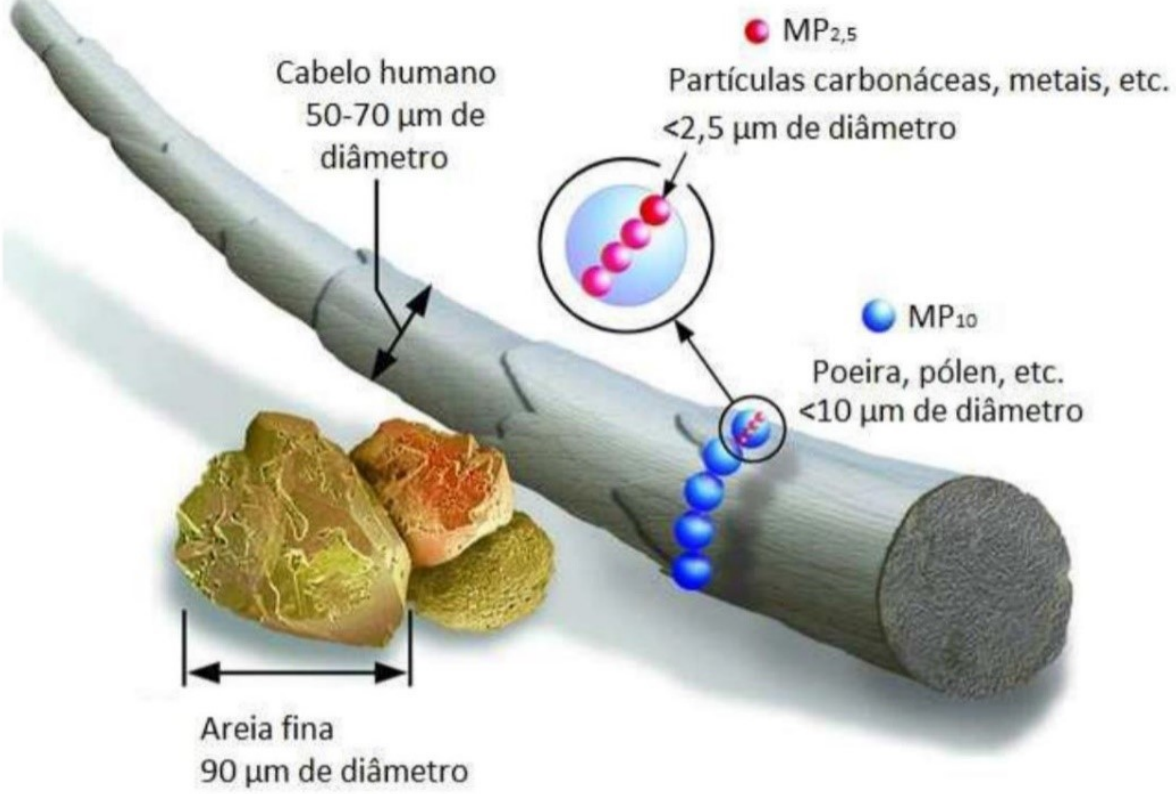

Fonte: Traduzido de U.S.EPA (2019)

A origem dessas partículas pode ser primária, em que são emitidas diretamente pela fonte, ou secundária, em que ocorrem reações químicas complexas, transformando outros poluentes em MP. Os poluentes que podem resultar em $\mathrm{MP}_{10}$ e $\mathrm{MP}_{2,5}$ são, normalmente, emitidos por usinas de energia, indústrias e automóveis. A Figura 1 enfatiza o quão pequenas essas partículas são, se comparadas com um fio de cabelo humano e grãos de areia, o que pode acarretar a formação de MP secundário em regiões distantes da fonte de emissão (U.S.EPA, 2019).

As fontes de emissão do MP ou dos componentes que o geram secundariamente podem ser de origem natural ou antrópica. A natureza libera partículas no ar em vários de seus processos, como a poeira de solos, erupções 
vulcânicas, incêndios naturais, spray marinho (partículas provenientes do oceano, carregadas pelo vento), aerossóis geológicos, pólens, esporos, bactérias, vírus, entre outros. Já a emissão por fontes antrópicas, a qual causa maiores impactos na saúde humana, engloba desde a agricultura, atividades industriais até processos de combustão, sendo a combustão a maior geradora de partículas bem pequenas e tóxicas, além da liberação de diversos outros componentes (BRITO; SODRÉ; ALMEIDA, 2018). Dentre as atividades industriais que mais contribuem para a formação de MP estão a indústria de cimento, centrais elétricas, entre outras. O tamanho das partículas, segundo a U.S.EPA (2019), está diretamente relacionado com o potencial de risco causado à saúde humana. $O \mathrm{MP}_{10}$ pode chegar ao pulmão, ou até na corrente sanguínea, porém pode ser eliminado pelo corpo humano mais facilmente. Já o $\mathrm{MP}_{2,5}$ apresenta maiores riscos à saúde, pois consegue chegar com mais facilidade nos sistemas respiratório e cardiovascular do corpo humano. Essa exposição pode levar à morte prematura por doenças relacionadas ao pulmão e coração, assim como ataques cardíacos, asma, deficiências de funcionamento do pulmão e batimentos cardíacos alterados, irritações nas vias respiratórias, entre outros. Inclusive, pessoas que já possuem alguma doença cardiorrespiratória são mais propensas a sofrer danos maiores com a inalação de MP (U.S.EPA, 2019). A quantificação da emissão desses poluentes atmosféricos, bem como de seu impacto potencial na saúde humana, está relacionada com o crescimento dos estudos referentes à área de Avaliação do Ciclo de vida (ACV). ACV é uma técnica que mensura os possíveis impactos relacionados ao ciclo de vida de determinado produto ou serviço, englobando todas as suas etapas de produção e utilização (IBICT, 2019). A implementação da ACV no Brasil depende de normas para que os procedimentos tomados sigam um padrão, o qual evita que haja discrepâncias entre cada análise de ACV e a existência de conflitos. A Organização Internacional de Normalização (ISO) está envolvida com ACV desde 1994, padronizando os métodos e procedimentos. Essa padronização ocorre por meio de duas normas: a ISO 14040, que consiste na técnica e princípios de ACV (NBR ISO 14040, 2009), e a ISO 14044, que abrange seus requisitos e diretrizes (NBR ISO 14044, 2009). Além das normas apresentadas pela ISO, a Comissão Europeia também proporciona suporte, a fim de garantir a qualidade de uma ACV. Esse suporte é feito por meio do Manual ILCD (do inglês, International Reference Life Cycle Data System handbook), o qual apresenta detalhadamente como deve ser realizada uma ACV da melhor forma possível (EC-JRC, 2011). A ACV é dividida em quatro etapas: definição do objetivo e escopo; análise do Inventário do Ciclo de Vida (ICV); Avaliação de Impacto do Ciclo de Vida (AICV); e interpretação. A análise dos impactos causados é definida na etapa de AICV, a qual relaciona cada fase do ciclo de vida proposta no ICV com os impactos potenciais presentes naquela fase, por meio de fatores de caracterização $(\mathrm{FC})$, os quais são obtidos em modelos de caracterização para determinadas categorias de impacto (NBR ISO 14040, 2009). As categorias de impacto abrangem diversas áreas, tais como o impacto em recursos bióticos e abióticos, meio ambiente (poluição do ar, água e solo) e saúde humana. Algumas dessas categorias são: acidificação; toxicidade humana; ecotoxicidade; efeito estufa; formação de material particulado (foco deste trabalho); entre outras (UNEP/SETAC, 2016).

O FC, para a categoria de material particulado, segue um caminho ambiental similar para MP primário ou secundário, sendo esse caminho dividido em outros fatores. Esses fatores são apresentados na Figura 2, a qual mostra a cadeia de 
causa-efeito, relacionando a exposição ao $\mathrm{MP}_{2,5}$ com os efeitos na saúde humana por meio da AICV.

Figura 2 - Cadeia de causa e efeito por exposição ao $\mathrm{MP}_{2,5}$ na AICV

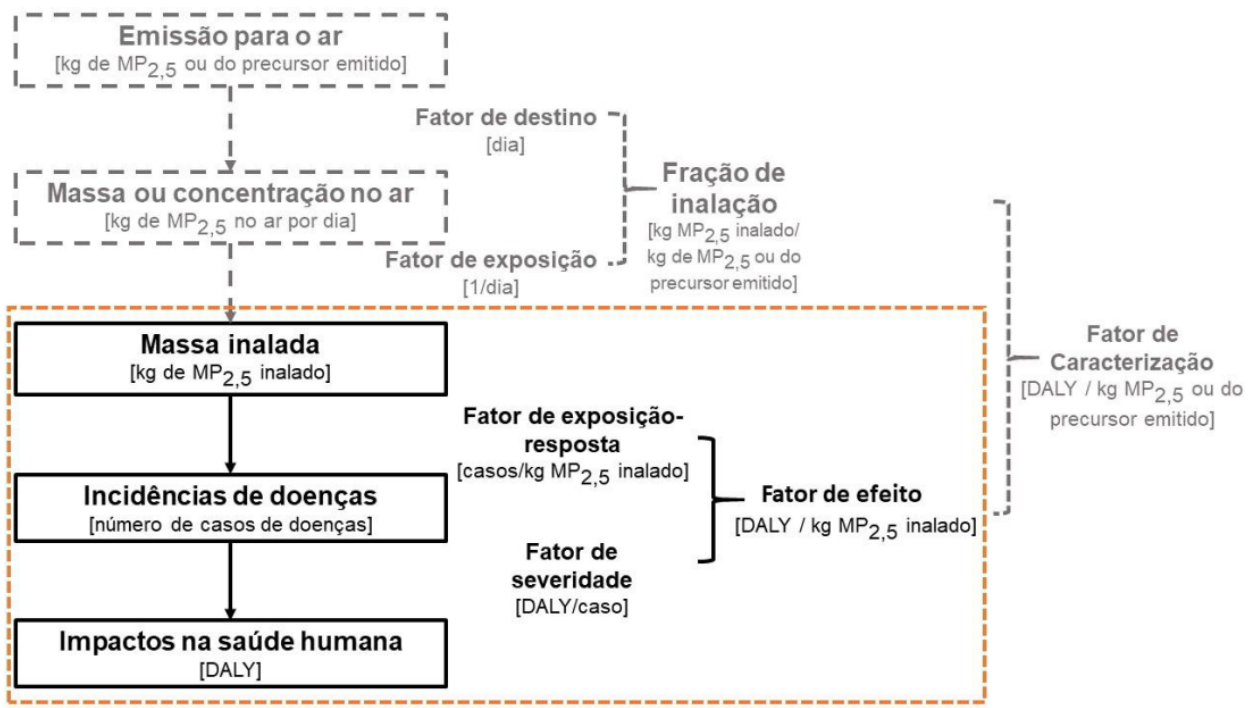

Fonte: Traduzido de UNEP/SETAC (2016)

A Figura 2 ilustra o caminho utilizado para chegar no FC pra a categoria formação de material particulado. A parte destacada compõe o fator de efeito, foco deste estudo. Primeiro, há uma quantidade de substância emitida no ar, medida em $\mathrm{kg}$ de $\mathrm{MP}_{2,5}$ ou precursor emitido $\left(\mathrm{NO}_{x}, \mathrm{SO}_{2}\right.$, amônia - $\mathrm{NH}_{3}$ e/ou Compostos Orgânicos Voláteis - COV). Em cada situação, há uma concentração dessas substâncias emitidas que permanece no are pode reagir e formar $\mathrm{MP}_{2,5}$ (kg de $\mathrm{MP}_{2,5}$ por dia). Relacionando essas duas quantidades, obtém-se o fator de destino, medido em dias. Dentro dessa concentração no ar, apenas uma parte é inalada ( $\mathrm{kg}$ de $\mathrm{MP}_{2,5}$ inalado) que, relacionada com a concentração diária, resulta no fator de exposição (1/dia). A cada quantidade inalada, apenas uma fração resulta em incidência de doenças (e, relacionando-a com a fração inalada, obtémse o fator de exposição resposta, medido em casos por $\mathrm{kg} \mathrm{de} \mathrm{MP}_{2,5}$ inalado. Por fim, dessas incidências, analisa-se qual à gravidade dos problemas causados, denominados de anos de vidas ajustados por incapacidade (DALY - do inglês, Disability-Ajusted Life Years), que, relacionado com a incidência de doenças, forma o fator de severidade, medido em DALY por caso. Relacionando os fatores, obtêm-se:

- Fator de inalação ( $\mathrm{kg}$ de $\mathrm{MP}_{2,5}$ inalado por $\mathrm{kg}$ de $\mathrm{MP}_{2,5}$ ou precursor emitido): relação do fator de destino com o fator de exposição;

- Fator de efeito (DALY por $\mathrm{kg} \mathrm{MP}_{2,5}$ inalado): relação do fator de exposição-resposta com o fator de severidade.

Finalmente, multiplicando-se os fatores de inalação (iF) e de efeito (EF), obtém-se o fator de caracterização (DALY por $\mathrm{kg} \mathrm{MP}_{2,5}$ ou precursor emitido), dado pela Equação (1), de acordo com UNEP/SETAC (2016):

$$
F C=i F \times E F
$$


A análise dos FC, priorizando o fator de efeito, envolve dar foco aos modelos utilizados como base para o cálculo dos fatores de exposição-resposta e de severidade. Na categoria de MP, os estudos já existentes abrangem Europa, EUA e Japão, países que possuem uma realidade diferente do Brasil, como características climáticas, socioeconômicas e regionais. Neste sentido, a categoria de impacto formação de material particulado vem sendo estudada no Brasil pela Rede de Pesquisa em Avaliação de Impacto do Ciclo de Vida (RAICV), criada em 2014 (ALMEIDA NETO; LINS; ALVARENGA, 2016, UGAYA et al., 2016). A RAICV padroniza o sistema de regionalização de modelos de caracterização para as diferentes categorias de impacto, objetivando analisar, adaptar, recomendar e desenvolver métodos de AICV mais adequados ao contexto brasileiro. A criação da RAICV foi justamente devido à falta de modelos adequados para se utilizar no Brasil (ALMEIDA NETO; LINS; ALVARENGA, 2016, UGAYA et al., 2016). Neste sentido, a análise de modelos de caracterização existentes para a categoria de formação de material particulado tem a finalidade de recomendar o modelo mais adequado para utilização no contexto brasileiro. Essa análise foi feita por meio da divisão do FC em outros dois fatores que originam o seu cálculo: o fator de inalação e o fator de efeito, sendo esse último, o foco deste trabalho. A análise de forma separada destes fatores traz uma avaliação mais profunda nos modelos de caracterização, possibilitando uma melhor adaptação à realidade nacional. Assim, conhecer as características de atividades industriais no contexto brasileiro é importante, visando uma futura regionalização dos modelos estudados, tornando as pesquisas de AICV brasileiras cada vez melhores. Neste trabalho, intenciona-se buscar dados e informações nos modelos de caracterização que são voltados ao fator de efeito, como as consequências na saúde humana, os anos de coleta desses dados, quais as substâncias poluentes e quais as regiões que o modelo considera em seus cálculos do fator de efeito. Posteriormente, esses modelos serão pontuados de acordo com critérios e subcritérios de avaliação, a fim de compará-los e recomendar o de maior pontuação, representando o mais adequado a ser utilizado no contexto brasileiro.

\section{METODOLOGIA}

Os modelos a serem avaliados neste trabalho foram previamente selecionados e são apresentados no Quadro 1. Esses modelos foram escolhidos devido à sua recomendação por Huschild e Huijbregts (2015) no livro LCA Compendium, com a inclusão dos modelos UNEP/SETAC (2016) e Van Zelm (2016), por serem mais atuais. Todos os modelos selecionados são reconhecidos pela comunidade científica. 
Quadro 1 - Classificação da resolução espacial dos fatores de efeito para material particulado dos modelos recomendados por Hauschild e Hujibregt (2015), mais UNEP/SETAC (2016) e Van Zelm et al. (2016)

\begin{tabular}{|c|c|c|}
\hline Modelos de caracterização & Resolução espacial & Método de AICV \\
\hline $\begin{array}{c}\text { Gronlund et al. (2014); } \\
\text { Humbert et al. (2011) }\end{array}$ & Continental & Impact World+ \\
\hline Humbert (2009) & Continental & ReCiPe \\
\hline $\begin{array}{c}\text { Goedkoop et al. (2009); } \\
\text { Van Zelm et al. (2008) }\end{array}$ & Europa \\
\hline $\begin{array}{c}\text { UNEP/SETAC (2016) } \\
\text { Jolliet et al. (2003), Itsubo e } \\
\text { Inaba (2012) }\end{array}$ & $\begin{array}{c}\text { Europa, América do Sul, } \\
\text { América do Norte e Ásia }\end{array}$ & IMPACrica do Norte \\
\hline $\begin{array}{c}\text { Bare (2003), Bare et al. } \\
\text { (2011) }\end{array}$ & $\begin{array}{c}\text { Nacional (EUA dividido por } \\
\text { estados) }\end{array}$ & TRACl 2/TRACI 1 \\
\hline $\begin{array}{c}\text { Van Zelm et al. (2016) } \\
\text { Global (56 regiões } \\
\text { emissoras e 56 regiões } \\
\text { receptoras) }\end{array}$ & - \\
\hline
\end{tabular}

Fonte: Adaptado de Hauschild e Hujibregt (2015) e Santos (2018)

Os modelos apresentados no Quadro 1 foram avaliados de acordo com os critérios e subcritérios apresentados no Quadro 2, a fim de compará-los e, futuramente, pontuá-los para recomendar o mais adequado para ser utilizado no contexto brasileiro. Os critérios levados em consideração nessa análise foram previamente definidos pela RAICV.

Quadro 2 - Critérios analisados nos modelos de caracterização para o fator de efeito

\begin{tabular}{|c|c|c|c|c|c|}
\hline \multirow{2}{*}{\multicolumn{2}{|c|}{$\begin{array}{l}\text { Critérios } \\
\text { Critério } 1 \text { - Escopo (Avaliação final) }\end{array}$}} & \multicolumn{4}{|c|}{ Níveis de classificação } \\
\hline & & Alta & Moderada & Baixa & $\begin{array}{l}\text { NA (Não se } \\
\text { aplica) }\end{array}$ \\
\hline 1.1 & Abrangência do escopo de aplicação & \multicolumn{4}{|c|}{ Global/Continental/Regional } \\
\hline 1.1 .1 & Nível de diferenciação espacial & \multicolumn{4}{|c|}{ Regiões consideradas no modelo } \\
\hline 1.1 .2 & Resolução temporal & \multicolumn{4}{|c|}{$\begin{array}{l}\text { Anos considerados nos dados coletados de efeitos na } \\
\text { saúde humana }\end{array}$} \\
\hline 1.1.3 & $\begin{array}{l}\text { Efeitos na saúde humana (health } \\
\text { endpoints) }\end{array}$ & \multicolumn{4}{|c|}{$\begin{array}{l}\text { Quantos e quais consequências na saúde foram } \\
\text { consideradas }\end{array}$} \\
\hline 1.1.4 & $\begin{array}{l}\text { Em nível de fluxos elementares } \\
\text { considerados }\end{array}$ & \multicolumn{4}{|c|}{$\begin{array}{l}\text { Quais as substâncias consideadas na formação de } \\
\text { material particulado }\end{array}$} \\
\hline 1.1.4.1 & $\begin{array}{l}\text { Ano de coleta das concentrações de } \\
\text { poluentes }\end{array}$ & \multicolumn{4}{|c|}{$\begin{array}{l}\text { Quais os anos considerados nas coletas das } \\
\text { concentrações de poluentes }\end{array}$} \\
\hline \multicolumn{2}{|c|}{ Critério 2 - Robustez científica (Avaliação final) } & Alta & Moderada & Baixa & NA \\
\hline 2.1 & Método de AICV ou comunidade científica & \multicolumn{2}{|l|}{ Sim } & \multicolumn{2}{|l|}{ Não } \\
\hline 2.1 .2 & Apresentação de cadeia de causa e efeito & \multicolumn{2}{|l|}{ Sim } & \multicolumn{2}{|l|}{ Não } \\
\hline 2.2 & Transparência e acessibilidade & \multicolumn{2}{|l|}{ Sim } & \multicolumn{2}{|l|}{ Não } \\
\hline 2.2 .1 & Clareza das equações & \multicolumn{2}{|l|}{ Sim } & \multicolumn{2}{|l|}{ Não } \\
\hline 2.1 .2 & Clareza das variáveis & \multicolumn{2}{|l|}{ Sim } & \multicolumn{2}{|l|}{ Não } \\
\hline \multicolumn{2}{|c|}{$\begin{array}{l}\text { Critério } 3 \text { - Fatores de Caracterização nacionais } \\
\text { (Avaliação final) }\end{array}$} & Alta & Moderada & Baixa & NA \\
\hline 3.1 & Possui FC para o Brasil & \multicolumn{2}{|l|}{ Sim } & \multicolumn{2}{|l|}{$\mathrm{Nao}$} \\
\hline 3.1 .1 & Arquétipos considerados & \multicolumn{4}{|c|}{$\begin{array}{l}\text { Quais os ambientes de emissão e formação de MP } \\
\text { considerados }\end{array}$} \\
\hline 3.1 .2 & FC apropriado para o contexto nacional & \multicolumn{2}{|l|}{ Sim } & Não & Não se aplica \\
\hline
\end{tabular}

Fonte: Adaptado de Santos et al. (2018)

Alguns dos itens apresentados no Quadro 2 foram modificados do quadro utilizado previamente por Santos et al. (2018), devido à separação da análise do FC em fator de Os critérios e subcritérios apresentados no Quadro 2 foram 
adotados em 2014, durante o II Workshop Internacional de ACV: AICV, realizado em Curitiba, PR. A definição desses critérios foi baseada nos critérios do ILCD Handbook (2011), juntamente com a classificação de Mendes (2013). Somente o Critério 3 - Fatores de Caracterização nacionais foi exclusivamente definido pela RAICV. Esses critérios e subcritérios são explicados a seguir, com base em Girotto (2018) e Santos (2018), com foco no fator de efeito.

CRITÉRIO 1 - ESCOPO: avalia a completeza do modelo de caracterização, analisando a abrangência de cada aspecto apresentado nos subcritérios (Alta/Moderada/Baixa/NA-Não se aplica).

1.1.1 Nível de diferenciação espacial: apresenta as regiões consideradas no modelo.

1.1.2 Resolução temporal: avalia os anos da coleta de dados dos efeitos na saúde humana considerados.

1.1.3 Efeitos na saúde humana (health endpoints): apresenta quantas e quais as consequências na saúde humana consideradas.

1.1.4 Em nível de fluxos elementares considerados: apresenta quais são as substâncias poluentes consideradas na emissão e formação de MP para o fator de efeito (MP primário, secundário e substâncias precursoras).

1.1.4.1 Ano de coleta das concentrações de poluentes: apresenta o(s) anos(s) da coleta dos dados das concentrações de cada substância poluente para o fator de efeito.

CRITÉRIO 2 - ROBUSTEZ CIENTÍFICA: avalia qual o nível transparência, clareza e acessibilidade dos dados utilizados no modelo de caracterização (Alta/Moderada/Baixa/NA-Não se aplica).

2.1 Método AICV ou comunidade científica: avalia se o modelo faz parte de algum método de AICV ou é reconhecido pela comunidade científica.

2.1.1 Apresentação da cadeia causa e efeito: aponta se o modelo apresenta a cadeia de causa e efeito ou não.

2.1 Transparência e acessibilidade: avalia se os documentos e as informações apresentadas são acessíveis e de fácil entendimento, sem deixar falhas de interpretação.

2.2.1 Clareza das equações: avalia se as equações envolvidas no cálculo do fator de efeito possuem clareza (Sim/Não).

2.2.2 Clareza das variáveis: avalia se as variáveis presentes nas equações do modelo são de fácil acesso e entendimento (Sim/Não).

CRITÉRIO 3 - FATORES DE CARACTERIZAÇÃO NACIONAIS: avalia se existe fator de caracterização para o Brasil e, se sim, qual o nível que os fatores de caracterização apresentados no modelo representam a realidade do país (Alta/Moderada/Baixa/NA-Não se aplica). 
3.1 Possuir fator de caracterização para o Brasil: avalia se o modelo apresenta um fator de caracterização específico para o Brasil ou não.

3.1.1 Arquétipos considerados: apresenta quais os ambientes de emissão e formação de MP são considerados no Brasil.

3.1.2 Fator de caracterização apropriado para o contexto nacional: avalia se o FC apresentado pelo modelo em questão é adequado para ser utilizado no Brasil ou não. Se sim, indica que o modelo retrata de forma abrangente e mais completa a categoria de formação do material particulado no Brasil. Caso contrário, o modelo possui um FC para o Brasil, porém não representa as suas condições adequadamente.

\section{RESULTADOS E DISCUSSÕES}

Os modelos de caracterização UNEP/SETAC (2016), Itsubo e Inaba (2012) e Jolliet (2003), bem como, seus respectivos métodos foram analisados de acordo com os critérios e subcritérios apresentados no Quadro 2. Esta análise é apresentada no Quadro 3.

Quadro 3 - Modelos de caracterização analisados

\begin{tabular}{|c|c|c|c|c|}
\hline \multirow{3}{*}{\multicolumn{2}{|c|}{$\begin{array}{l}\text { Critérios } \\
\text { Critério } 1 \text { - Escopo } \\
\text { (Avaliação final) }\end{array}$}} & \multicolumn{3}{|l|}{ Modelos de caracterização } \\
\hline & & \multirow{2}{*}{$\begin{array}{l}\text { UNEP/SETAC (2016) } \\
\text { Moderada }\end{array}$} & \multirow{2}{*}{$\begin{array}{l}\text { Itsubo e Inaba (2012) } \\
\text { Baixa }\end{array}$} & \multirow{2}{*}{$\begin{array}{l}\text { Jolliet (2003) } \\
\text { Baixa }\end{array}$} \\
\hline & & & & \\
\hline 1.1 & $\begin{array}{l}\text { Abrangência do } \\
\text { escopo de } \\
\text { aplicação }\end{array}$ & Global & Nacional & Continental \\
\hline 1.1 .1 & $\begin{array}{l}\text { Nível de } \\
\text { diferenciação } \\
\text { espacial }\end{array}$ & $\begin{array}{l}\text { Europa, América do Sul, } \\
\text { América do Norte e Ásia }\end{array}$ & Japão (8 regiões) & $\begin{array}{l}\text { Europa e América do } \\
\text { Norte }\end{array}$ \\
\hline 1.1.2 & $\begin{array}{l}\text { Resolução } \\
\text { temporal }\end{array}$ & 1990,2005 e 2010 & $1987,1995,1996$ e2002 & 1980, 1995 e 1996 \\
\hline 1.1 .3 & $\begin{array}{l}\text { Efeitos na saúde } \\
\text { humana (health } \\
\text { endpoints) }\end{array}$ & $\begin{array}{l}\text { Doença } \\
\text { cerebrovascular, doença } \\
\text { pulmonar, câncer de } \\
\text { pulmão, entre outros }\end{array}$ & $\begin{array}{l}\text { Mortes, incapacidades e } \\
\text { doenças respiratórias }\end{array}$ & $\begin{array}{l}\text { Sintomas respiratórios } \\
\text { inferiores, mortalidade } \\
\text { por doenças crônicas } \\
\text { obstrutivas } \\
\text { pulmonares, entre } \\
\text { outros }\end{array}$ \\
\hline 1.1 .4 & $\begin{array}{l}\text { Em nível de } \\
\text { fluxos } \\
\text { elementares } \\
\text { considerados }\end{array}$ & $\mathrm{MP}_{10}$, TSP e MP 2,5 & $\mathrm{MP}_{10}$, e $\mathrm{MP}_{2,5}$ & $\mathrm{MP}_{10}, \mathrm{TSP}$ e $\mathrm{MP}_{2,5}$ \\
\hline 1.1.4.1 & $\begin{array}{l}\text { Ano de coleta das } \\
\text { concentrações de } \\
\text { poluentes }\end{array}$ & 1990,2005 e 2010 & $1987,1995,1996$ e 2002 & 1980, 1995 e 1996 \\
\hline
\end{tabular}

Fonte: Autoria própria (2019) 
Quadro 3 - Modelos de caracterização analisados (Continuação)

\begin{tabular}{|c|c|c|c|c|}
\hline \multicolumn{2}{|c|}{$\begin{array}{l}\text { Critério } 2 \text { - Robustez } \\
\text { científica (Avaliação final) }\end{array}$} & \multirow{2}{*}{$\begin{array}{l}\text { Alta } \\
\text { Sim }\end{array}$} & \multirow{2}{*}{$\begin{array}{l}\text { Baixa } \\
\text { Sim }\end{array}$} & \multirow{2}{*}{$\begin{array}{l}\text { Baixa } \\
\text { Sim }\end{array}$} \\
\hline 2.1 & $\begin{array}{l}\text { Método de AICV } \\
\text { ou comunidade } \\
\text { científica }\end{array}$ & & & \\
\hline 2.1 .2 & $\begin{array}{l}\text { Apresentação de } \\
\text { cadeia de causa e } \\
\text { efeito }\end{array}$ & Sim & Sim & Sim \\
\hline 2.2 & $\begin{array}{l}\text { Transparência e } \\
\text { acessibilidade }\end{array}$ & Sim & Não & Não \\
\hline 2.2 .1 & $\begin{array}{l}\text { Clareza das } \\
\text { equações }\end{array}$ & Sim & Não & Não \\
\hline 2.2 .2 & $\begin{array}{l}\text { Clareza das } \\
\text { variáveis }\end{array}$ & Sim & Não & Não \\
\hline \multicolumn{2}{|c|}{$\begin{array}{l}\text { Critério } 3 \text { - Fatores de } \\
\text { Caracterização nacionais } \\
\text { (Avaliação final) }\end{array}$} & Baixa & Baixa & Baixa \\
\hline 3.1 & $\begin{array}{l}\text { Possui FC para o } \\
\text { Brasil }\end{array}$ & $\begin{array}{l}\text { Não (mas considera } \\
\text { dados do Brasil)* }\end{array}$ & Não & Não \\
\hline 3.1 .1 & $\begin{array}{l}\text { Arquétipos } \\
\text { considerados }\end{array}$ & Não se aplica & Não se aplica & Não se aplica \\
\hline 3.1 .2 & $\begin{array}{l}\text { FC apropriado } \\
\text { para o contexto } \\
\text { nacional }\end{array}$ & Não & Não & Não \\
\hline
\end{tabular}

*Apresenta FC por arquétipo e não por região geográfica

O Quadro 3 apresenta detalhadamente a análise realizada para os modelos de caracterização, nos quais foram buscadas informações a respeito dos critérios e subcritérios para o fator de efeito de cada um desses modelos. Pode-se perceber também que os dados relacionados ao contexto brasileiro são escassos, pois a maioria dos modelos abrangem apenas regiões da Europa e EUA, com algumas exceções, como América do Sul no geral, e Japão. A internacionalidade dos modelos de caracterização implica em dados regionais, de efeitos na saúde, substâncias e métodos de AICV considerados diferentes do Brasil, o que pode acarretar em resultados não tão próximos à realidade desejada. A separação dos fatores de efeito e de inalação possibilita uma avaliação mais minuciosa destes modelos, trazendo os dados mais precisos do cálculo do fator de caracterização em cada caso. A RAICV publicou o Relatório de Recomendações de Modelos de Avaliação de Impacto para o Contexto Brasileiro, a fim de uso e da regionalização de FC para as categorias de impacto de acidificação, eutrofização, recursos bióticos e abióticos, método de contabilidade de recursos, escassez hídrica e serviços ecossistêmicos. Esse relatório resulta em uma contribuição para os estudos de AICV nacionais, além de ser uma base para futuras regionalizações de outras categorias de impacto, assim como a de formação de MP (RAICV, 2019).

Como exemplo, a categoria de escassez hídrica, cuja definição envolve o desequilíbrio entre a disponibilidade e demanda de água de uma determinada região, de acordo com as características dessa região. A regionalização de um entre os modelos selecionados e avaliados para essa categoria objetivou o desenvolvimento de meios de avaliar os impactos de produtos e processos relacionados à escassez hídrica da região abordada (RAICV, 2019).

A metodologia utilizada segue um caminho semelhante ao abordado neste trabalho, englobando a identificação dos modelos, seguido de uma avaliação individual de cada um, passando por uma análise quantitativa e uma 
recomendação do modelo mais apropriado para a regionalização no contexto brasileiro. No caso da categoria de escassez hídrica, mais de um modelo foi recomendado, devido a diferentes situações, em que há certos modelos mais apropriados para cada uma (RAICV, 2019). Neste sentido, a categoria de MP também poderá ter mais de um modelo recomendado, devido à análise separada dos fatores de efeito e de inalação, sendo que cada análise pode chegar a um modelo específico e não necessariamente igual ao outro. Além disso, também há a possibilidade de nenhum dos modelos serem adequados o suficiente para a sua regionalização, o que também pôde ser observado em Santos (2018), pois os modelos analisados apresentaram dados insuficientes para concluir em uma recomendação mais próxima do desejado. Para a categoria de MP, os modelos de AICV analisados em Santos (2018) apresentaram essa falta de bons dados relacionados ao contexto brasileiro. Esse fato também se estende para o restante dos modelos que foram analisados neste trabalho.

\section{CONCLUSÃO}

Com os trabalhos previamente analisados, pode-se concluir que, são poucas as informações a respeito do cenário brasileiro consideradas nestes modelos de caracterização para a categoria de impacto formação de material particulado. Alguns dos modelos previstos serão analisados posteriormente. Após a sua avaliação e classificação, estes serão avaliados quantitativamente, permitindo a recomendação do modelo mais adequado a ser utilizado interinamente nos estudos de ACV no Brasil. A importância de se obter um modelo para ser utilizado no contexto brasileiro se deve à falta de dados disponíveis, atualmente, para realizar uma AICV considerando esta categoria de impacto. Ao ser recomendado um modelo interinamente para o Brasil, espera-se avançar cientificamente em estudos de AICV e, também, possibilitar resultados mais próximos à realidade nacional. A separação dos fatores de efeito e inalação intenciona trazer resultados mais precisos para a classificação de cada modelo. Por fim, o modelo recomendado para um dos fatores pode não ser necessariamente o mesmo para o outro, o que também resulta em fator de caracterização mais próximo à realidade do Brasil. É sabido que a atividade industrial é uma das principais causas de impactos ao meio ambiente, especialmente quanto à formação de MP. Assim, este trabalho apresentou resultados importantes rumo à produção sustentável, afinal, modelar os impactos para esta categoria de impacto tendo em vista a realidade brasileira é uma necessidade para que mais avanços científicos ocorram nesta direção e, assim, futuramente, as empresas possam utilizar tais modelos para estimar os danos ao meio ambiente que as suas atividades podem causar. 


\title{
Life cycle impact assessment: effect factors to particulate matter
}

\begin{abstract}
The industrial activity is one of the main causes of environmental impacts, especially related to particulate matter formation in the air. One way to consider this impacts is the use of Life Cycle Assessment (LCA) aims to analyze the life cycle involved in a product or process and connect the impacts caused in each stage of this cycle. This technique depends on four phases, one of them, the Life Cycle Impact Assessment (LCIA) phase is the focus of this work. In this phase, the impacts are related with each result from the Life Cycle Inventory $(\mathrm{LCl})$ by characterization models. These models aim to calculate a characterization factor (CF) that will measure the impact level in the existing impact categories. To particulate matter formation category, this work focus is to evaluate characterization models in order to identify the most appropriate to apply in Brazil. These models analysis was done by the CF division in intake fraction and effect factor, the last one being focus of this work. Three models were analyzed and the others will be analyzed after and all quantitatively evaluated, aiming to recommend the most suitable to be applied in Brazil. Thereby, it will allow a scientific breakthrough in LCIA researches, beyond the possibility of results more reliable and closer to the Brazilian reality.
\end{abstract}

KEYWORDS: Particulate matter. Characterization factors. Recommendation. Brazilian reality. LCIA. 
ALMEIDA NETO, J. A., LINS, I. de O., ALVARENGA, R. A. F. Recomendação de métodos de contabilização de recursos. In: CONGRESSO BRASILEIRO EM GESTÃO DO CICLO DE VIDA, 5. Fortaleza, 2016.

ASSOCIAÇÃO BRASILEIRA DE NORMAS TÉCNICAS (ABNT). NBR ISO 14040/2009: Gestão ambiental - Avaliação do ciclo de vida - Princípios e estrutura. Rio de Janeiro, 2009.

ASSOCIAÇÃO BRASILEIRA DE NORMAS TÉCNICAS (ABNT). NBR ISO 14044/2009: Gestão ambiental - Avaliação do ciclo de vida - Requisitos e orientações. Rio de Janeiro, 2009.

BRITO, G. F. S., SODRÉ, F. F., ALMEIDA, F. V. O Impacto do Material Particulado na Qualidade do Ar. Revista Virtual de Química. Brasília, 2018.

EC-JRC - European Commission Joint Research Centre. Recommendations for Life Cycle Impact Assessment in the European context: based on existing environmental impact assessment models and factors. ILCD handbook International Reference Life Cycle Data System, European Union EUR24571EN, 2011.

GIROTTO, S. B.F. T. Avaliação de modelos de caracterização de AICV para a categoria smog considerando o contexto brasileiro. 128 f. Dissertação (Mestrado em Ciências Ambientais) - Universidade do Estado de Santa Catarina, Santa Catarina, 2018.

HAUSCHILD, M. Z.; HUIJBREGTS, M. A. J. Life Cycle Impact Assessment. LCA Compendium - The Complete World of Life Cycle Assessment: Chapter 6: Particulate Matter Formation, [S.I.], p.97-114, 2015. crossref

INSTITUTO BRASILEIRO DE INFORMAÇÃO EM CIÊNCIA E TECNOLOGIA (IBICT). Histórico da ACV. Brasília, 2019. Disponível em <http://acv.ibict.br/acv/historicoda-acv/>. Acesso em: 22 maio 2019.

MENDES, N. C. Métodos e modelos de caracterização para a Avaliação de Impacto do Ciclo de Vida: análise e subsídio para a aplicação no Brasil. 2013. 149f. Dissertação (Mestrado em Engenharia de Produção) - Escola de Engenharia de São Carlos, Universidade de São Paulo, São Carlos.

Rede de Pesquisa de Avaliação do Impacto do Ciclo de Vida - RAICV.

Recomendação de modelos de avaliação de impacto do ciclo de vida para o 
contexto brasileiro. 2019. 165 p. Organização: Cássia Maria Lie Ugaya, José Adolfo de Almeida Neto e Maria Cléa Brito de Figueiredo. Brasília, DF: Ibict.

SANTOS, P. N. A. Avaliação de modelos de caracterização de AICV para a categoria material particulado no contexto brasileiro. 2018. 85 f. Trabalho de Conclusão de Curso (Graduação em Engenharia Química) - Universidade Tecnológica Federal do Paraná, Paraná.

SANTOS, P. N. A. et al. Avaliação de modelos para a categoria de impacto formação de material particulado em avaliação de impacto do ciclo de vida (AICV) para o Brasil. In: CONGRESSO BRASILEIRO SOBRE GESTÃO DO CICLO DE VIDA, 6. Brasília, 2018.

UGAYA, C. M. L. et al. Critérios para recomendar modelos de caracterização de AICV no Brasil. In: CONGRESSO BRASILEIRO EM GESTÃO DO CICLO DE VIDA, 5. Fortaleza, 2016.

UNITED NATIONS ENVIRONMENT PROGRAMME (UNEP); SOCIETY OF ENVIRONMENTAL TOXICOLOGY AND CHEMISTRY (SETAC). Global Guidance for Life Cycle Impact Assessment Indicators. Life Cycle Iniciative, v. 1, 2016.

UNITED STATES ENVIRONMENTAL PROTECTION AGENCY (U.S.EPA). Particulate Matter (PM) Basics. 2019. Disponível em: <https://www.epa.gov/pmpollution/particulate-matter-pm-basics\#PM>. Acesso em: 15 maio 2019.

UNITED STATES ENVIRONMENTAL PROTECTION AGENCY (U.S.EPA). Particle Pollution and Your Health, US Environmental Protection Agency, set. 2003. Disponível em: <https://nepis.epa.gov/Exe/ZyPDF.cgi?Dockey=P1001EX6.txt>. Acesso em: 15 maio 2019.

AN ZELM, R. et al. (2016) Regionalized life cycle impact assessment of air pollution on the global scale: Damage to human health and vegetation. Atmospheric Environment. crossref

WORLD HEALTH ORGANIZATION (OMS). WHO Air quality guidelines for particulate matter, ozone, nitrogen, dioxide and sulfur dioxide. 2005. Disponível em:<https://apps.who.int/iris/bitstream/handle/10665/69477/WHO_SDE_PHE_ OEH_06.02_eng.pdf?sequence=1>. Acesso em: 13 maio 2019. 
Recebido: 04 Dez. 2019

Aprovado: 31 Dez. 2019

DOI: $10.3895 /$ gi.v15n4.11881

Como citar:

CHELI, G. R. et al. Avaliação de impacto do ciclo de vida: fatores de efeito para material particulado. $\mathbf{R}$.

Gest. Industr., Ponta Grossa, v. 15, n. 4, p. 14-27, Out./Dez. 2019. Disponível em:

http://periodicos.utfpr.edu.br/revistagi. Acesso em: 2019.

Correspondência:

Gabriela Roiko Cheli

Universidade Tecnológica Federal do Paraná, Ponta Grossa, Paraná, Brasil.

Direito autoral: Este artigo está licenciado sob os termos da Licença Creative Commons-Atribuição 4.0

Internacional.

(c) (1) 